
Military Technical College Kobry El-Kobbah
Cairo, Egypt

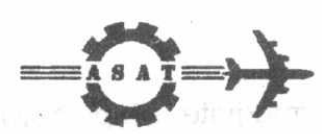

$10^{\text {th }}$ International Conference

On Aerospace Sciences\&

Aviation Technology

\title{
DEVELOPMENT OF A CLASSIFIER FOR MFSK SIGNALS OVER TIME VARYING CORRELATED FADING CHANNELS UNDER CLASS- A IMPULSIVE NOISE
}

\author{
EL-MAHDY` A. E
}

\section{ABSTRACT}

In a recent work [1], we developed and analyzed a classifier for multiple M-ary Frequency Shift Keying (MFSK) signals that traveled over a static frequency nonselective Rayleigh fading channel and are contaminated with additive white Gaussian noise. In this paper, we extend the classifier to the case in which the MFSK signals traveled over a time varying flat correlated fading channel and contaminated with impulsive noise. Specifically, the considered impulsive noise is the Middelton's class A noise model. The classifier is based on approximating the likelihood function (LF) of the received signal. We use complex envelope representation of the signals and noise to derive the likelihood function. The Karhunen-Loeve expansion is used to have a more precise description of the fading process. Simulation experiments are illustrated to evaluate the performance of the classifier and to validate the theoretical developments.

\section{KEY WORDS}

Signal Intercept, Modulation Recognition, and Fading channels with impulsive noise 


\section{INTRODUCTION}

Signal classification is an intermediate step between signal interception and demodulation. It plays an important role in both military and civilian applications due to its capability of placing several receivers in one universal receiver. In $\bullet$ military applications, the foremost purpose of electronic surveillance is the threat recognition, by comparing the signal characteristics of intercepted emitters against a catalog of characteristics or signal sorting parameters. This is desirable for a number of reasons inciuding signal confirmation, interference identification, suitable jamming signal selection, and proper demodulator selection (to prevent damage of the signal information content). In civilian application, the task of monitoring electromagnetic signal transmission in the RF spectrum has become a priority in broadcasting controi especially with the increasing demand for radio communications. Another task is the supervision of admitted wireless stations, to determine whether they obey the limits of their operation parameters, as well as to detect non-licensed transmitters.

Literature includes various articles in the area of MFSK signal classification [1]-[4]. For example, El-Mahdy and Namazi [1] developed and analyzed classifier for multiple additive MFSK signals traveled over a time invariant frequency non-selective fading channel. The classifier was derived for both synchronous and asynchronous waveforms. Two cases were considered in [1]; the first one was concerned with the signals that have identical modulation type (for example BFSK or QFSK). The second case deals with the signals with different modulation type. Bessel and Charles [2] explored a general framework that theoretically links the higher-order correlation domain with statistical decision theory. Then, they applied this technique to the problem of classification of MFSK signals when contaminated with additive white Gaussian noise. They presented two types of MFSK classifiers: channelized and non-channelized classifiers. It was claimed that the non-channelized classifiers are immune to imperfect knowledge of exact frequency locations. They extended their technique to the classification of asynchronous MFSK Signals [3] in which the signal arrival time is unknown. Nandi and Azzouz [4] presented two algorithms for classification of communication signals. The first one utilized the decision theoretic approach in which a global procedure for analog and digitally modulated signal recognition is proposed. The second algorithm was based on the artificial neural network. It was found that good performance is obtained by the algorithm when the signal to noise ratio $(\mathrm{SNR})=15 \mathrm{~dB}$.

As presented, the researchers have not focused on the problem of classification of MFSK signals traveled over a correlated fading channel. Moreover, they assumed that the received signal is contaminated with white Gaussian noise. Non of them focus on developing classifiers under non-Gaussian impulsive noise. In this paper, we develop a classifier for M-ary frequency shift keying signals received through a time varying flat correlated fading channel. The signal is assumed to be contaminated with impulsive non-Gaussian noise, specifically with Middelton's class A impulsive noise model is a more practical model, which arises, in digital communication systems.

The general model of the MFSK signal considered in this study has the form [1] 


$$
S(t)=\operatorname{Re}\left\{\sqrt{E_{s}} \sum_{n} \exp \left\{j\left(2 \pi f^{(n)} t+\theta^{(n)}\right\} \times p\left(t-n T_{s}\right) e^{j \omega_{c} t}\right\}\right.
$$

where $E_{s}$ is the signal energy, $\theta^{(n)}$ is a set of random variables that is uniformly distributed over the interval $[-\pi, \pi], n$ is the symbol number, $p(t)$ is the standard unit pulse of duration $T_{s}$, and $f^{(n)}$ is a set of independent identically distributed (i.i.d) discrete random variables (r.v's), the elements of which are uniformly distributed on $\{-B / 2, B / 2]$, where $B$ is the bandwidth of the MFSK signal. The considered MFSK signal arises in many digital communication systems. It represents the signal models in many applications such as radar and sonar of unknown Doppler returns and spread spectrum communications of fast frequency hopping kind. Also, this signal is useful for modeling phase-modulated signals, which tend to occupy few orthogonal dimensions at all time, under lack of the exact knowledge of the carrier frequency. It is required to classify these transmissions, that is to determine $M$ for each received signal, where $M$ indicates the modulation type $(M=2$ corresponds to $B F S K, M=4$ corresponds to QFSK and so on).

This paper is organized as follows. In section II, the model and the features of the class A impulsive noise model are summarized. In section III, we mathematically formulate the classification problem for MFSK signals. The mathematical derivation of the decision rule is presented in this section. In section IV, simulation experiments are presented to demonstrate the performance of the classification scheme. Finally we present the summary and conclusions in section $\mathrm{V}$.

\section{CLASS A IMPULSIVE NOISE MODEL}

In order to determine the classifier for the MFSK signals, a mathematical model for the impulsive noise is required. Middleton's canonical impulsive noise is often used as a statistical-physical model of the impulsive radio noise. In this model, according to the relation between the durations of the noise impulses and the spectral bandwidth at the receiver, the impulsive noise is classified into three general classes: class A, B, and C. Through some experimental measurements, the actual impulsive noise supports the Middleton's canonical class A model. The class A impulsive noise has a density function $f(n)$ that consists of an infinite weighted sum of Gaussian densities with decreasing weights and increasing variances. Specifically, the probability density function of the normalized (unit power) noise is given by $[5, \mathrm{pp}$. 86]:

$$
f(n)=\sum_{m=0}^{\infty} \frac{e^{-A} A^{m}}{m ! \sqrt{2 \pi \sigma_{m}^{2}}} \exp \left(-\frac{n^{2}}{2 \sigma_{m}^{2}}\right)
$$

where the parameter $A$ is called the impulsive index: it is the product of the received average number of impulses per unit time and the duration of an impulse. This parameter defines the impulsiveness of the noise. For small $A$, the noise becomes more impulsive and for larger $A$, the statistical characteristics of the class $A$ impulsive noise approach those of Gaussian noise. The variances $\sigma_{m}^{2}$ are related to the physical parameters and are given by: 


$$
\sigma_{m}^{2}=\frac{(m / A)+\Gamma}{1+\Gamma}, \quad m=0,1,2, \ldots
$$

where $\Gamma$ is the ratio of the mean power of the Gaussian noise component to the nonGaussian impuisive noise component. An approximation to the model in (2) can be obtained by limiting the sum to the first $Z$ terms only. Small value of $Z$ is found to be sufficient to give excellent approximation to the noise probability density functions.

\section{MATHEMATICAL FORMULATION OF MFSK CLASSIFIER}

In this section, we derive the decision rule for the classification of MFSK signals that traveled over flat time varying correlated fading channel and contaminated with class A impulsive noise described in section II. Consider the complex envelope of a received observation, $\tilde{y}(t)$, which consists of the per-symbol complex envelope of MFSK signal received through time varying flat correlated fading channel plus the complex envelope of a white Gaussian noise:

$$
\tilde{y}(t)=\tilde{a}(t) \tilde{s}(t)+\widetilde{w}(t)
$$

where $\widetilde{w}(t)$ is the complex envelope of the class A impulsive non-Gaussian noise process and $\tilde{s}(t)$ is the per-symbol complex envelope of the MFSK signal. In addition, $\widetilde{a}(t)$ is complex Gaussian process with autocorrelation function $R_{\tilde{a}}(\tau)$. The fading rate is slow such that the fading complex parameter $\widetilde{a}(t)$ is practically constant over the signal symbol duration $T_{s}$. The per-symbol complex envelope of the signal $\widetilde{s}(t)$, is given by [3]

$$
\tilde{s}(t)=\sqrt{E} e^{j(2 \pi f t+\theta)}
$$

where $\{f\}$ is the frequency of the signal which is assumed to be a discrete random variable uniformly distributed on $[-B / 2, B / 2], B$ is the bandwidth of the signal defined as $B=\frac{M}{2 T_{s}}$ and $\theta$ is the phase of the signal which is uniformly distributed over the interval $[-\pi, \pi]$. The fading process $\tilde{a}(t)$ may be expanded using the Karhunen-Loeve expansion [6, Ch. 9], [7, Ch. 3] as

$$
\tilde{a}(t)=\sum_{k=1}^{\infty} \widetilde{a}_{k} \phi_{k}(t), \quad t \in[a, b]
$$

where $\widetilde{a}_{k} ; k=1,2, . ., \infty$ are zero-mean independent Gaussian complex variables with variances $\lambda_{k}=E\left\{\left|\widetilde{a}_{k}\right|^{2}\right\}$ and $\phi_{k}(t) ; \quad k=1,2, . ., \infty$ are orthonormal eigen functions which solve the homogeneous integral equation

$$
\int_{a}^{b} R_{\tilde{a}}(t-\tau) \phi_{k}(\tau) d \tau=\lambda_{k} \phi_{k}(t), \quad t \in[a, b]
$$


The summation in (5) can be truncated to th : first $K$ terms. If we order the eigen functions so that $\lambda_{1} \geq \lambda_{2} \geq \ldots$, then (5) can be written as

$$
\tilde{a}(t)=\sum_{k=1}^{K} \widetilde{a}_{k} \varphi_{k}(t), \quad t \in[a, b]
$$

The relevant likelihood function (LF) of $\tilde{y}(t)$ with respect to the random parameters $\tilde{a}(t)$ and $f$ is given by:

$$
\Delta[\tilde{y}(t) ; \widetilde{a}(t), f]=\sum_{m=0}^{\infty} \frac{e^{-A} A^{m}}{m ! \sqrt{2 \pi \sigma_{m}^{2}}} \exp \left\{\frac{-1}{2 \sigma_{m}^{2}} \int_{0}^{T_{s}}\left(|\widetilde{a}(t) \widetilde{s}(t)|^{2}-2 \operatorname{Re}\left(\tilde{y}(t) \tilde{a}^{*}(t) \tilde{s}^{*}(t)\right)\right)\right\} d t
$$

where $T_{s}$ is the symbol duration of the MFSK signal. By substitution of (7) and (4) into (8), we have:

$$
\begin{aligned}
& \Delta\left[\tilde{y}(t) ; \tilde{a}_{k}, f\right]=\sum_{m=0}^{\infty} \frac{e^{-A} A^{m}}{m ! \sqrt{2 \pi \sigma_{m}^{2}}} \exp \left\{-\frac{1}{2 \sigma_{m}^{2}}\left(\int_{0}^{T_{s_{1}}}\left|\sum_{k=1}^{K} \tilde{a}_{k} \phi_{k}(t) \widetilde{s}(t)\right|^{2} d t\right.\right. \\
&\left.\left.-2 \sqrt{E_{s}} \sum_{k=1}^{K} \operatorname{Re}\left(\int_{0}^{T_{s_{t}}} \tilde{y}(t) \phi_{k}(t) \tilde{a}_{k}^{*} e^{-j(2 \pi f t+\theta)} d t\right)\right)\right\}
\end{aligned}
$$

The first term inside the exponential of (9) can be expanded as $\left.\int_{0}^{T_{x_{1}}}\left|\sum_{k=1}^{K} \widetilde{a}_{k} \phi_{k}(t) \widetilde{s}(t)\right|^{2} d t=\sum_{k=1}^{K} \int_{0}^{T_{s_{t}}}\left|\tilde{a}_{k} \phi_{k}(t) \widetilde{s}(t)\right|^{2} d t-\sum_{k} \sum_{j=j} \int_{0}^{T_{s}} \operatorname{Re}\left\{\tilde{a}_{k} \phi_{k}(t) \widetilde{s}(t) \tilde{a}_{j}^{*} \phi_{j}(t) \tilde{s}^{*}(t)\right\} d t\right\}(10)$

Using the orthonormal property of the basis waveforms, the second term in (10) is vanished and (10) becomes

$$
\int_{0}^{T_{31}}\left|\sum_{k=1}^{K} \tilde{a}_{k} \phi_{k}(t) \tilde{s}(t)\right|^{2} d t=\gamma_{s} \sum_{k=1}^{K}\left|\tilde{a}_{k}\right|^{2}
$$

where $\gamma_{s}=E_{s} T_{s}$ is the per-symbol signal to noise ratio (since the noise is normalized to have unit power). It is clear that this term is a hypothesis independent term and can be dropped without affecting the decision of the classifier, then, the LF in (9) can be written as

$$
\Delta\left[\tilde{y}(t) ; \widetilde{a}_{k}, f\right]=\sum_{m=0}^{\infty} \frac{e^{-A} A^{m}}{m ! \sqrt{2 \pi \sigma_{m}^{2}}} \exp \left\{\frac{2 \sqrt{\gamma_{s}}}{2 \sigma_{m}^{2}}\left(\sum_{k=1}^{K}\left|Y_{k}(f)\right|\left|\tilde{a}_{k}\right| \cos (\theta)\right)\right.
$$

where $Y_{k}(f)=\frac{1}{\sqrt{T_{s}}} \int_{0}^{T_{s}} \tilde{y}(t) \phi_{k}(t) e^{-j 2 \pi f t} d t$ is the normalized Fourier transform of $\tilde{y}(t) \phi_{k}(t)$ within the symbol duration of the signal $T_{s}$ and $E_{s}$ is the energy of the signal. For $N$ independent and identically distributed (i.i.d) symbols, the LF, $\Delta_{n}\left[\tilde{y}(t) ; \tilde{a}_{k}, f\right]=\prod_{n=1}^{N} \Delta\left[\tilde{y}(t) ; \tilde{a}_{k}, f\right]$, is given by 


$$
\begin{aligned}
& \Delta_{n}\left[\tilde{y}(t) ; \widetilde{a}_{k}, f\right]=\left(\sum_{m=0}^{\infty} \frac{e^{-A} A^{m}}{m ! \sqrt{2 \pi \sigma_{m}^{2}}}\right)^{N} \\
& \quad \exp \left\{\sum_{n=1}^{N} \ln E_{\theta, a_{k}, f}\left[\exp \left(\frac{\sqrt{\gamma_{s}}}{\sigma_{m}^{2}} \sum_{k=1}^{K}\left|\widetilde{a}_{k}\right|\left|Y_{k}^{(n)}(f)\right| \cos (\theta)\right)\right]\right.
\end{aligned}
$$

where $Y_{k}^{(n)}(f)=\frac{1}{T_{s}} \int_{(n-1) r_{s}}^{n T_{t}} \widetilde{y}(t) \phi_{k}(t) e^{-j 2 \pi f t} d t$ is the normalized Fourier transform of $\tilde{y}(t) \phi_{k}(t)$ within the $n$-th symbol duration of the signal and $E_{\theta, a_{k}, f}$ denotes the expectation with respect to the random variables $\theta, a_{k}$ and $f$ respectively. Expanding the inner exponentials using Taylor's series representation $\left(e^{x}=1+x+0.5 x^{2}+\ldots\right)$; truncated to the third term, then taking the expectation with respect to $\theta$ and $a_{k}$, we have

$$
\begin{aligned}
& \Delta_{n}[\widetilde{y}(t), f] \approx\left(\sum_{m=0}^{\infty} \frac{e^{-A} A^{m i}}{m ! \sqrt{2 \pi \sigma_{m}^{2}}}\right)^{N} \\
& \exp \left\{\sum_{n=1}^{N} \sum_{k=1}^{K} \ln E_{f}\left(1+\left(\frac{\sqrt{\gamma_{s}}}{\sigma_{m}^{2}}\right)^{2} \lambda_{k}\left|Y_{k}^{(n)}(f)\right|^{2}\right)\right\}
\end{aligned}
$$

The $3^{\text {rd }}$ term truncation in the previous equation is performed to give a simple structure classification rule and also provide a good performance. Taking the average over the random variable $a_{i k}$ we have

$$
\Delta_{n}[\tilde{y}(t) ; f] \approx\left(\sum_{m=0}^{\infty} \frac{e^{-A} A^{m}}{m ! \sqrt{2 \pi \sigma_{m}^{2}}}\right)^{N} \exp \left\{\sum_{n=1}^{N} \sum_{k=1}^{K} \ln E_{f}\left(1+\left(\frac{\sqrt{\gamma_{s}}}{\sigma_{m}^{2}}\right)^{2} \lambda_{k}\left|Y_{k}^{(n)}(f)\right|^{2}\right)\right\}
$$

By taking the average over $f$, the likelihood function becomes

$$
\Delta_{n}[\widetilde{y}(t)] \approx\left(\sum_{m=0}^{\infty} \frac{e^{-A} A^{m}}{m ! \sqrt{2 \pi \sigma_{m}^{2}}}\right)^{N} \exp \left\{\sum_{n=1}^{N} \sum_{k=1}^{K} \ln \left[1+\frac{\lambda_{k}}{M}\left(\frac{\sqrt{\gamma_{s}}}{\sigma_{m}^{2}}\right)^{2}\left(\sum_{i=\frac{M}{2}}^{M / 2}\left|Y_{k, i}^{(n)}\right|^{2}\right)\right]\right\}
$$

where $Y_{k, m}^{(n)}=\frac{1}{T_{s}} \int_{(n-1) T_{s}}^{n T_{s}} \tilde{y}(t) \phi_{k}(t) e^{-j 2 \pi \frac{m}{2 T_{s} t}} d t$ is the normalized Fourier transform of $\widetilde{y}(t) \phi_{k}(t)$ at the $m$-th frequency location of the signal evaluated at the $n$-th symbol duration of that signal. For our assumption of small signal to noise ratio and using linear approximation of $\ln (1+x)$, the log-likelihood function (LLF) finally becomes 


$$
\Delta_{n}^{\prime}[\widetilde{y}(t)] \approx N \ln \left(\sum_{m=0}^{\infty} \frac{e^{-A} A^{m}}{m ! \sqrt{2 \pi \sigma_{m}^{2}}}\right)+\left(\frac{\sqrt{\gamma_{s}}}{\sigma_{m}^{2}}\right)^{2} \sum_{n=1}^{N} \sum_{k=1}^{K} \frac{\lambda_{k}}{M}\left(\sum_{q=\frac{M}{2}}^{M / 2}\left|Y_{k, q}^{(n)}\right|^{2}\right)
$$

where $Y_{k, q}^{(n)}=\frac{1}{T_{s}} \int_{(n-1) r_{s}}^{n T_{s}} \tilde{y}(t) \phi_{k}(t) e^{-j 2 \pi \frac{q}{2 T_{s} t}} d t$ is the normalized Fourier transform of $\tilde{y}(t) \phi_{k}(t)$ at the $m$-th frequency location of the signal evaluated at the $n$-th symbol duration of that signal. The proposed signal classifier under class $A$ impulsive noise is derived using the LLF given by (17). But, designing a classifier based on this LLF has great complications. A simplification of this LLF can be performed under the condition that the impulsive index $A$ is sufficiently small. Using this assumption, the infinite sum in (2) can be approximated by the maximum value of its first three terms [7]. According to this approximation, the pdf of the noise becomes:

$$
f(n)=\max _{m=0,1,2}\left[\frac{e^{-A} A^{m}}{m ! \sqrt{2 \pi \sigma_{m}^{2}}} \exp \left(-\frac{n^{2}}{2 \sigma_{m}^{2}}\right)\right]
$$

Then using (17) and (18), the simplified LLF can be written as

$$
\Delta_{\mathrm{n}}^{\prime}[\widetilde{\mathrm{y}}(\mathrm{t})]=\sum_{\mathrm{n}=1}^{N}\left(\max _{\mathrm{m}=0,1,2}\left[\ln \left(\frac{\mathrm{A}^{\mathrm{m}}}{\mathrm{m} ! \sigma_{\mathrm{m}}}\right)+\left(\frac{\sqrt{\gamma_{\mathrm{s}}}}{\sigma_{\mathrm{m}}^{2}}\right)^{2} \sum_{\mathrm{k}=1}^{\mathrm{K}} \frac{\lambda_{\mathrm{k}}}{\mathrm{M}}\left(\sum_{\mathrm{q}=\frac{\mathrm{-M}}{2}}^{\mathrm{M} / 2}\left|\mathrm{Y}_{\mathrm{k}, \mathrm{q}}^{(\mathrm{n})}\right|^{2}\right)\right]\right]
$$

The developed classifier is derived using the resulted LLF given by (19). In this case, it is assumed that the symbol duration of the signal is known and the received observation contains $M_{l}$ FSK signal under the hypothesis $H_{l}$ where $1=0,1,2, \ldots$. The symbol duration of the signal under the hypothesis $H_{l}$ is denoted by $T_{s, l}$. In the general case, we have a multiple hypotheses testing problem. Here, the problem is solved by deriving the decision rule, which determines the best decision among them. The decision rule is derived from the LLF equation for each hypothesis, which is derived from (19). In the following we will consider a binary hypotheses testing problem; extension to more hypotheses testing is straightforward. Suppose that we have two equally likely hypotheses: $H_{o}$ and $H_{1}$ corresponding to $\mathrm{Mo}$ and $\mathrm{M} 1$, respectively with $M 1<M o$. The binary test can be formulated as follows. Given Mo and $M 1$, the intent is to identify whether Mo or M1 is the modulation type of the received signal. From (19) it follows that the optimal decision rule is to compare the following expression to a threshold $\eta$, that is, 


$$
\begin{aligned}
\sum_{n=1}^{N}\left(\max _{m=0,1,2}\left[\ln \left(\frac{A^{m}}{m ! \sigma_{m}}\right)+\left(\frac{\sqrt{\gamma_{s}}}{\sigma_{m}^{2}}\right)^{2} \sum_{k=1}^{K} \frac{\lambda_{k}}{M_{1}}\left(\sum_{q=\frac{M_{1}}{2}}^{M_{1} / 2}\left|Y_{k, q, 1}^{(n)}\right|^{2}\right)\right]-\right. \\
\sum_{n=1}^{N}\left(\max _{m=0,1,2}\left[\ln \left(\frac{A^{m}}{m ! \sigma_{m}}\right)+\left(\frac{\sqrt{\gamma_{s}}}{\sigma_{m}^{2}}\right)^{2} \sum_{k=1}^{K} \frac{\lambda_{k}}{M_{0}}\left(\sum_{q=\frac{M_{0}}{2}}^{M_{0} / 2}\left|Y_{k, q, 0}^{(n)}\right|^{2}\right)\right]\right)_{\succ^{H_{1}}}^{\prec} \eta
\end{aligned}
$$

where

$$
Y_{k, q, l}^{(n)}=\frac{1}{T_{s}} \int_{n T_{s, l}}^{(n+1) T_{s, l}} \tilde{y}(t) \phi_{k}(t) e^{-j 2 \pi \frac{q}{2 T_{s, l}} d} d t ; \quad l=0,1
$$

$T_{s, l} ;(l=0,1)$ is the symbol duration of signal under hypothesis $/$ and $\eta$ is a threshoid level. The receiver announces $H_{1}$ (or $M_{1}$ FSK) when the threshold is exceeded and decides in favor of $H_{0}$ (or $M_{0}$ FSK) otherwise.

\section{SIMULATION EXPERIMENTS}

In this section we present the results of the simulation experiments. Experimental evaluation of the performance of the MFSK classifier is performed for the two hypotheses test; namely, the QFSK modulation corresponding to $M_{1}$ FSK and the 8FSK modulation corresponding to MoFSK. The autocorrelation function of the correlated fading channel is chosen to be $R_{\widetilde{a}}(\tau)=J_{0}\left(2 \pi f_{d} \tau\right)$ where $J_{o}(x)$ is the modified Bessel function of zero order. The parameters used in the simulation are: $M_{0}=8$ (i.e. 8-FSK), $M_{1}=4$ (i.e. QFSK), $f_{c}=500 \mathrm{kHz}$ (carrier frequency) and the fading rate is considered: $f_{d} T_{s}=0.02$. Extensive Monte carlo simulations are implemented to evaluate the performance of the classifier. The performance of the classifier is measured in terms of the probability of mis-classification $\left(P_{\mathrm{e}}\right)$ as a function of the signal to noise ratio (SNR). The effect of the variation of the impulsive index $(A)$ and the mean power ratio of Gaussian to impulsive noise $(\Gamma)$ on the performance of the classifier is studied. Also, the effect of the frequency offset on the performance of both ciassifiers is studied for different values of the parameters $A$ and $\Gamma$.

In Fig.1, the probability of mis-classification is evaluated by assuming that the mean power ratio of the Gaussian to the impulsive noise $\Gamma=0.001$ and varying the impulsive index $A$ between the values $0.001,0.01$, and 0.1 . The figure shows that as the impulsive index $A$ becomes smalier, the noise impulsiveness becomes stronger, thus causing larger performance degradation. In Fig.2, the impulsive index is assumed to be 1 , and the mean power ratio of the Gaussian to the impuisive noise varies in the range $[0.01,3]$. The results shows that when $A=1$ and $\Gamma$ becomes larger, the performance of the system is enhanced. This is because when $A=1$, an the impulsive noise occurs continuously, thus the impulsiveness becomes weaker arid weaker as $\Gamma$ becomes larger. 
To illustrate the performance of the synchronous decision rule against a carrier frequency offset between the transmitter and the classifier, we present Figure 3 which plots the probability of mis-classification for QFSK/8-FSK as a function of the frequency offset for different values of the impulsive index $A$. The figure shows that the probability of mis-classification increases as the frequency offset increases. It is clear from this figure that the performance of the decision rule is invariant to frequency offset over a wide range of frequencies. This range is increased as the impulsive index $A$ becomes larger, for example, at $A=0.0001$, this frequency range is up to $80 \mathrm{kHz}$ while at $A=1$, this range is extended to $135 \mathrm{kHz}$. This is because as $A$ increased, the impulsiveness become weaker and then this is implies increasing the classifier resistivety against frequency offset. When the carrier offset is large enough, part of the signal lies in the stop band of the noise limiting band pass filter, causing signal distortion. The probability of mis-classification gets worse as the signal distortion increases.

\section{CONCLUSIONS}

We developed a classifier for MFSK signals contaminated with class A impulsive noise and traveled over a time varying correlated flat fading channel. Karhunen-Loeve expansion has been used to expand the correlated fading channel random process. The approximated likelihood function of the MFSK is used to derive the decision rule for the classifier. The decision rule depends on the impulsive noise parameters. 


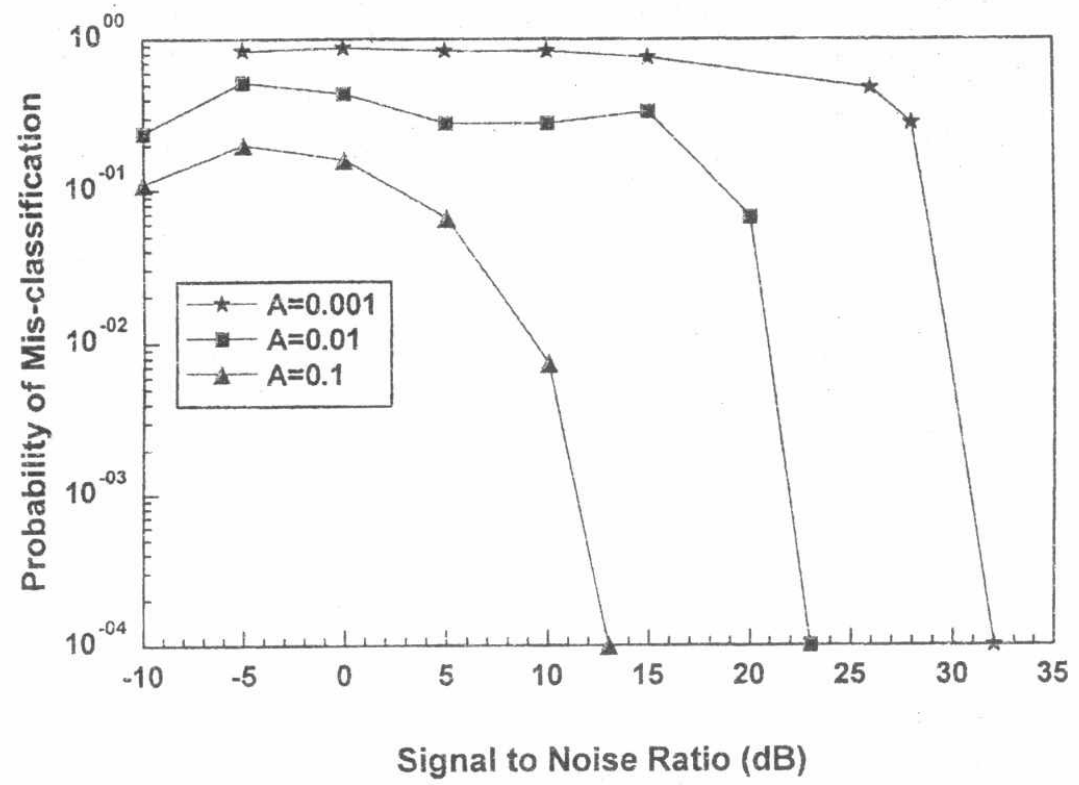

Fig. 1 : Probability of mis-classification versus $S N R$ for $A=0.001,0.01,0.1$, and $\Gamma=0.001$.

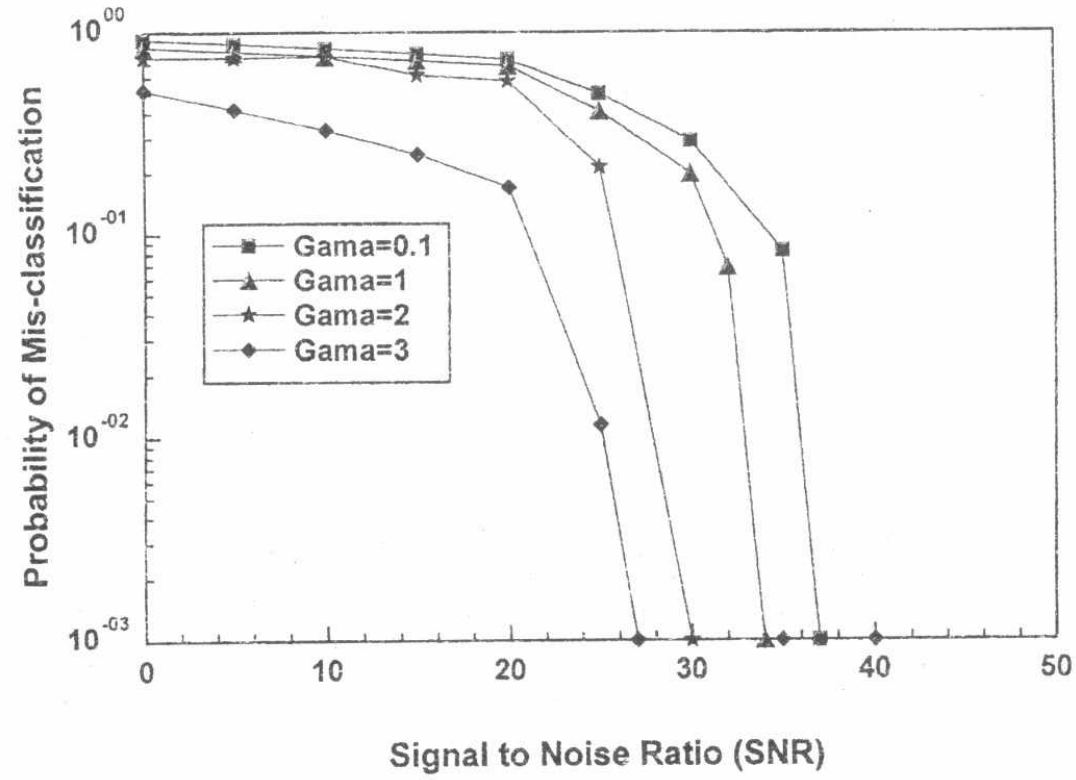

Fig. 2 : Probability of mis-classification versus $S N R$ for $A=1$ and $\Gamma=0.1, \Gamma=1$, and $\Gamma=2$. 


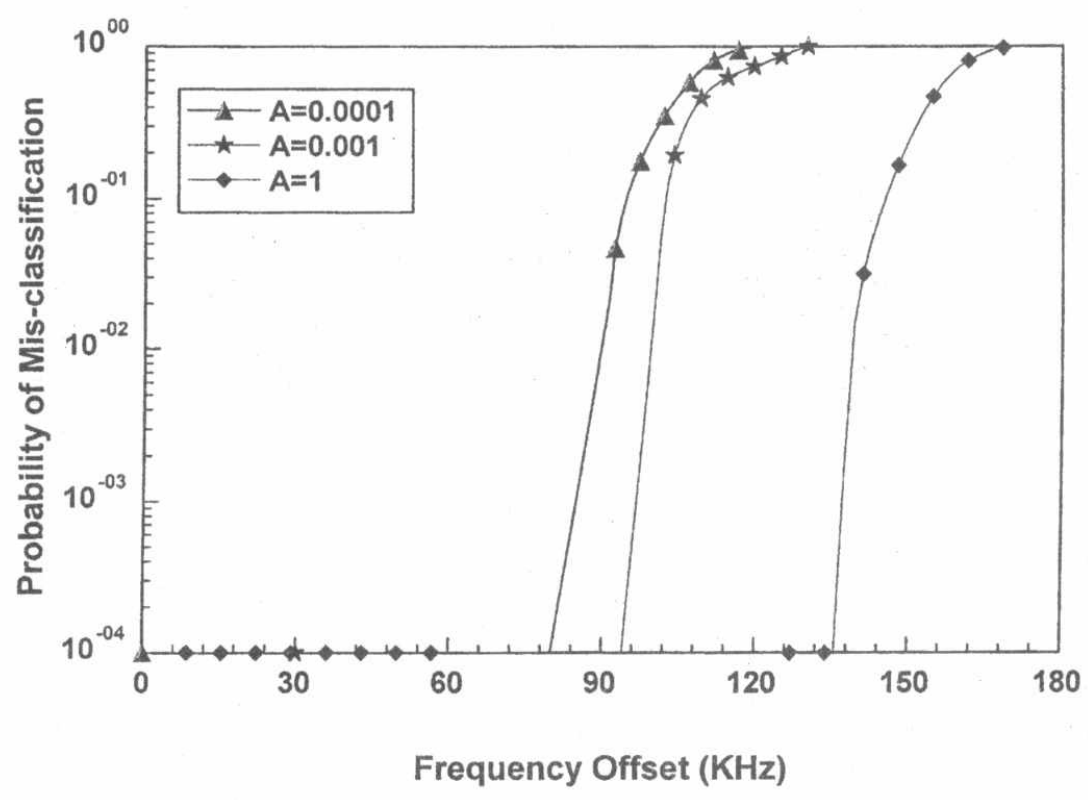

Fig. 3 : Probability of mis-classification Versus the frequency Offset for the synchronous classifier for different values of $A$ and for $\Gamma=0.01$ and $S N R=30 \mathrm{~dB}$. 


\section{References}

[1] Ahmed E. El_Mahdy, and Nader Namazi, "Classification of Multiple M-ary Frequency Shift Keying Signals Over Rayleigh Fading Channel", IEEE Trans.on Communications, Vol. 50, No. 6, June 2002.

[2] Bessel, F. and Charles, L.,"Higher-order Correlation Based Approach to Modulation Classification of Digitally Frequency-Modulated Signals", IEEE on Selected Areas in Communications, Vol. 13, No. 1, January 1995.

[3] Bessel, F. and Charles, L.,"Asynchronous Classification of MFSK Signals Using the Higher Order Correlation Domain", IEEE Trans.on Communications, Vol. 46, No. 4, April 1998.

[4] Nandi, A. and Azzouz, E., "Algorithms for Automatic Modulation Recognition of Communication Signals", IEEE Trans. On Communications, Vol. 46, No. 4, April1998.

[5] Whalen, A. D., Signal Detection in Non-Gaussian Noise. Dowden \& Culver, Inc., 1988.

[6] Whalen, A. D., Detection of Signals in Noise. New York : Academic, 1971.

[7] Van Trees, H. L., Detection, Estimation, and Modulation Theory, Part I, Wiley, 1968

[8] Shinichi, M., Masaaki, K. and Norihiko M., "Performance Analysis of QAM Systems Under Class A Impulsive Noise Environment", IEEE Trans. On Communications, Vol. 37, No. 2, May1995. 